\title{
MIL-101 (Fe) modified carbon paste electrode for the efficient simultaneous detection of hydroquinone and catechol
}

\author{
Mengting Zhu, Ruli Xu, Xueyang Wang, Jie Liu Qianli Zhang ${ }^{*}$, Jie Wei ${ }^{*}$ \\ School of chemistry and life Science, Suzhou University of Science and Technology, 1 Kerui Road, \\ Suzhou 215011, China \\ *E-mail: zqlmhb@163.com (Q. Zhang) ustsweijie@163.com (J. Wei)
}

Received: 14 August 2021 / Accepted: 27 September 2021 / Published: 10 November 2021

\begin{abstract}
Four Fe-based metal-organic frameworks (MIL-101(Fe), MIL-53(Fe), MIL-88(Fe), and MIL-100(Fe)) were synthesized using a solvothermal method, and further characterized by X-ray diffraction, Fourier transforms infrared spectroscopy, Scanning electron microscopy, and X-ray photoelectron spectroscopy. The synthesized Fe-MOFs were used to fabricate the modified carbon paste electrodes (CPE) for simultaneous detection of hydroquinone and catechol. Among the MILs (Fe) modified CPEs, MIL101(Fe)/CPE exhibited extraordinary electrochemical sensitivity because of MIL-101 (Fe)'s excellent conductivity. The oxidation potential separation of hydroquinone and catechol achieved $190 \mathrm{mV}$. Compared with CPE, the oxidation currents of hydroquinone and catechol at MIL-101/CPE were 10fold higher and the reduction currents were 5-fold higher. The linear range was $2-90 \mu \mathrm{M}$ for hydroquinone and $2-100 \mu \mathrm{M}$ for catechol, and the detection limit was $0.58 \mu \mathrm{M}$ and $0.62(\mathrm{~S} / \mathrm{N}=3) \mu \mathrm{M}$ for hydroquinone and catechol. Furthermore, the application of MIL-101 (Fe)/CPE has been validated in an actual river water sample with the satisfied recoveries of $90.21-102.36 \%$ and $91.11-102.19 \%$ for hydroquinone and catechol, respectively.
\end{abstract}

Keywords: Electrochemical detection, MILs (Fe), Hydroquinone and catechol, Modified carbon paste electrode, Differential pulse voltammetry

\section{FULL TEXT}

(C) 2021 The Authors. Published by ESG (www.electrochemsci.org). This article is an open access article distributed under the terms and conditions of the Creative Commons Attribution license (http://creativecommons.org/licenses/by/4.0/). 